\title{
Harmonic Resonance Enhanced Second-Harmonic Generation in the Monolayer $\mathrm{WS}_{2}$-Ag Nanocavity
}

\author{
Xiaobo Han ${ }^{1}$, Kai Wang ${ }^{2, *}$, Patrick D. Persaud ${ }^{3}$, Xiangyuan Xing ${ }^{2}$, Weiwei Liu ${ }^{2}$, Hua \\ Long $^{2}$, Fang Li $^{1}$, Bing Wang ${ }^{2}$, Mahi R. Singh ${ }^{3, *}$ and Peixiang Lu ${ }^{1,2,4, *}$ \\ ${ }^{1}$ Hubei Key Laboratory of Optical Information and Pattern Recognition, Wuhan Institute of \\ Technology, Wuhan 430205, China
}

${ }^{2}$ Wuhan National Laboratory for Optoelectronics and School of Physics, Huazhong University of Science and Technology, Wuhan 430074, China

${ }^{3}$ Department of Physics and Astronomy, The University of Western Ontario, London N6G 3K7, Canada

${ }^{4}$ CAS Center for Excellence in Ultra-intense Laser Science, Shanghai 201800, China

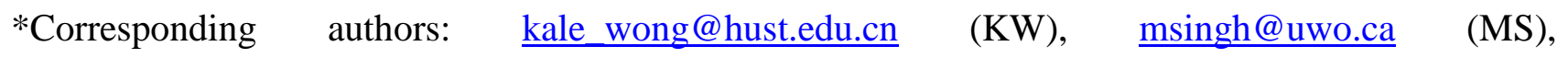
lupeixiang@hust.edu.cn (PXL) 


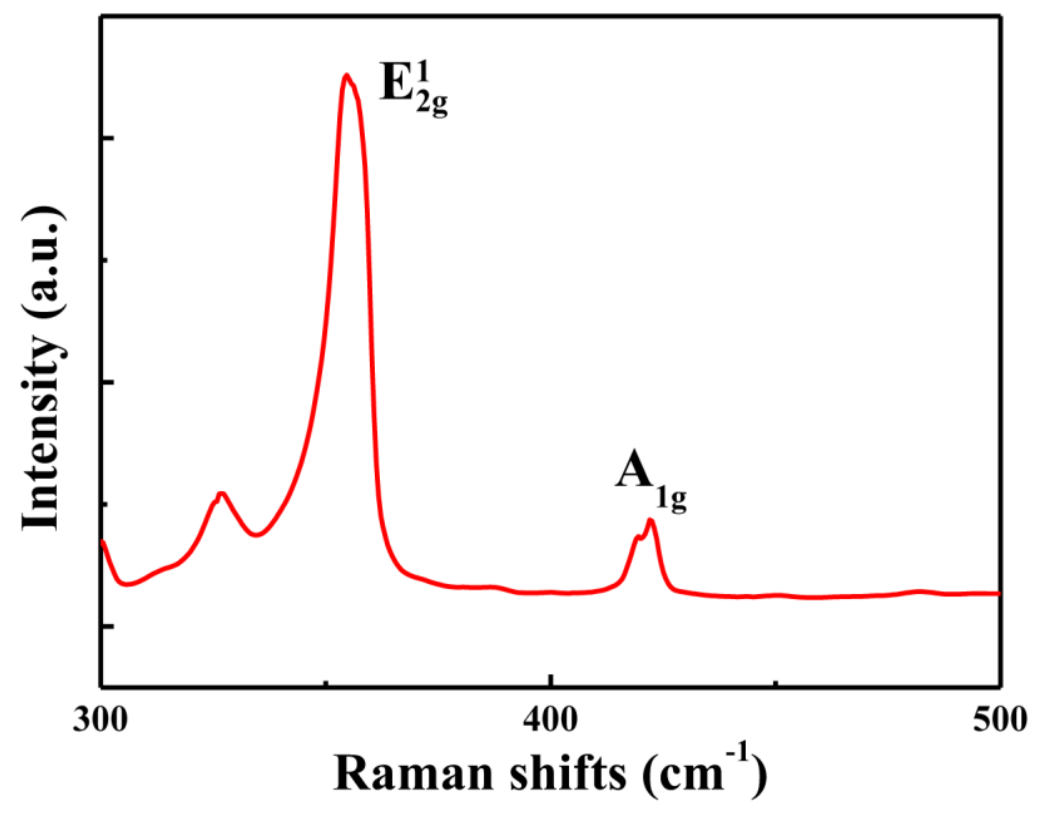

Figure S1. The Raman spectrum of the $\mathrm{WS}_{2}$ flake taken at $300 \mathrm{~K}$ with an excitation wavelength of $532 \mathrm{~nm}$. The frequency of these two modes is around $356 \mathrm{~cm}^{-1}$ and $419 \mathrm{~cm}^{-1}$, respectively. The energy separation of these two modes and characteristic of SHG signal confirmed the single layer nature of our sample. ${ }^{1}$ 


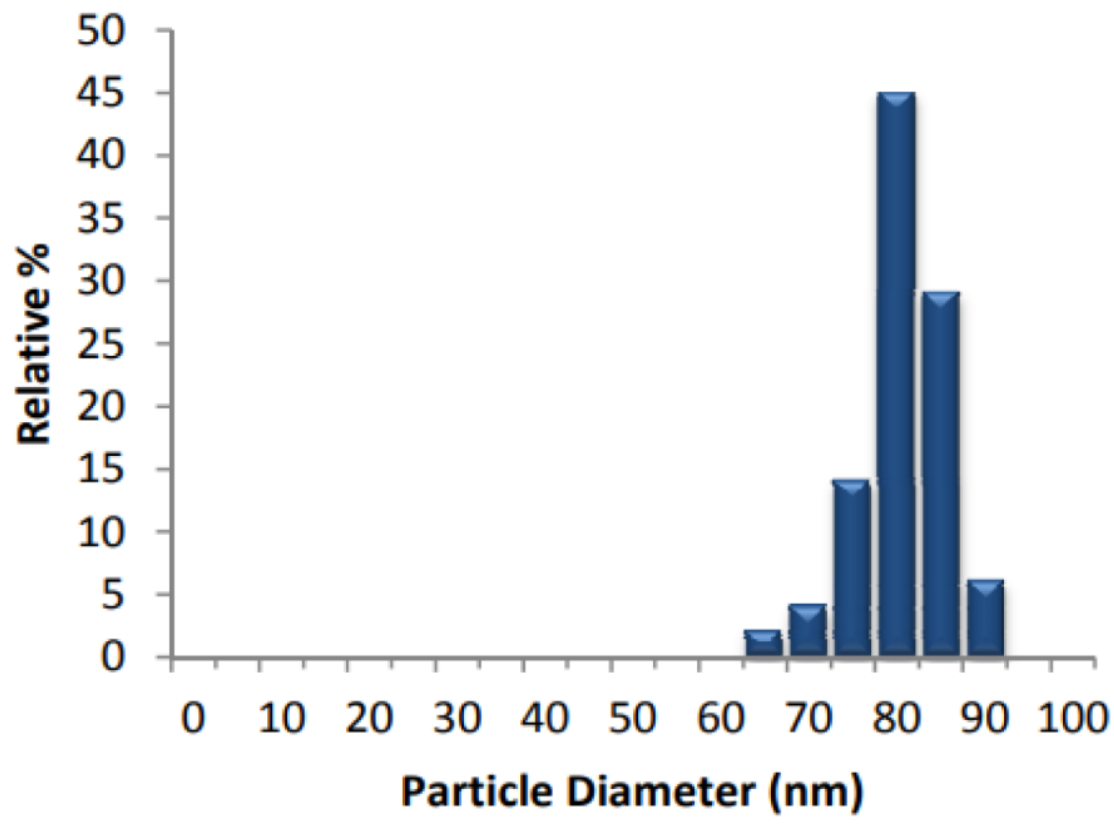

Figure S2. The size distribution of the silver nanocubes. (Referenced by nanoComposix https://tools.nanocomposix.com:48/cdn/coa/Silver/Nanocubes/KJW1966_75_nm_Ag_Cubes_C_of_ A-For\%20Web.pdf?2442214) 


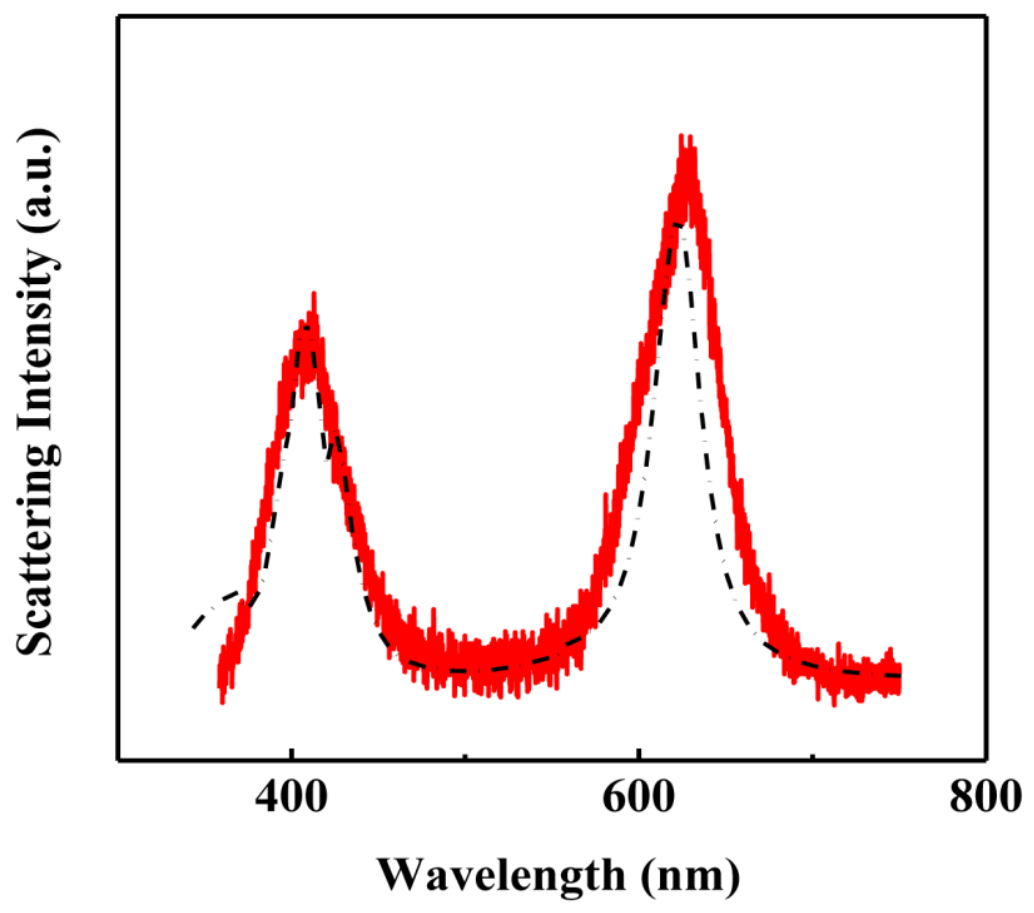

Figure S3. Simulated (black dashed curve) and measured (red curve) scattering results of a single nanocavity with two SPPR wavelengths due to the dipole and quadrupole resonance modes. 


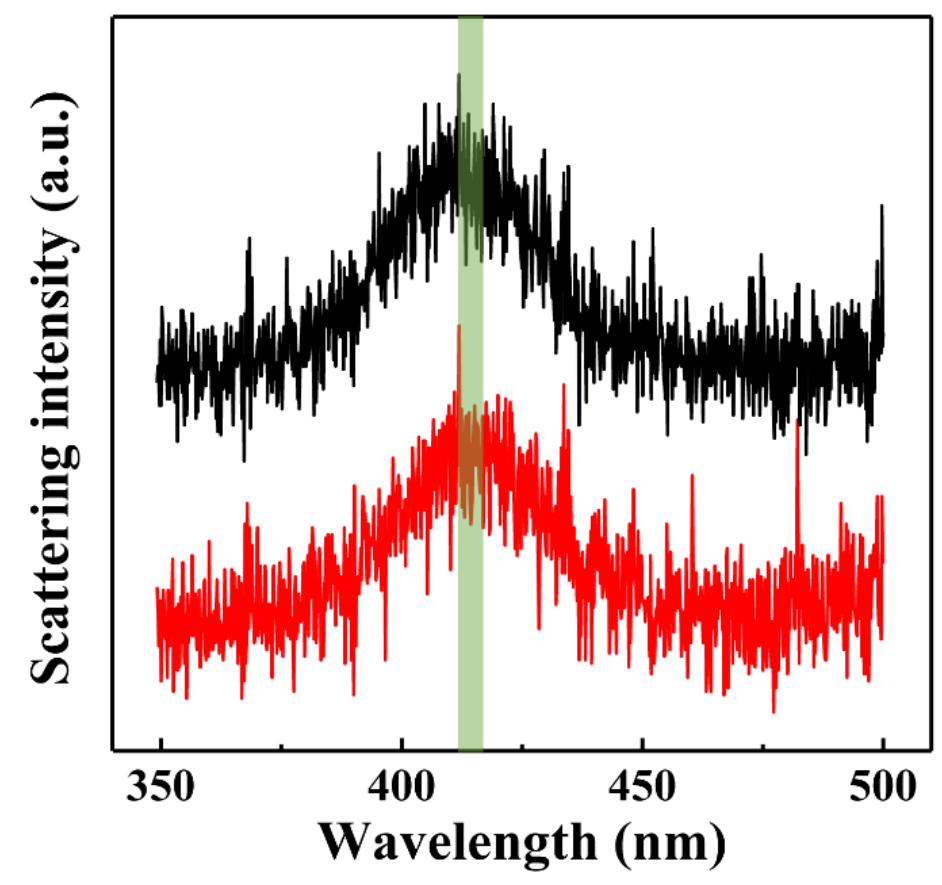

Figure S4. Scattering spectra of the nanohybrid before (black line) and after (red line) SHG measurement. The LSPR peaks before and after SHG measurement are 412 and $415 \mathrm{~nm}$, respectively. The scattering peak is slightly red-shifted $(\sim \mathrm{nm})$ or even undistinguishable. It indicates that the $\mathrm{Ag}$ nanocubes are not damaged after the SHG measurement. 


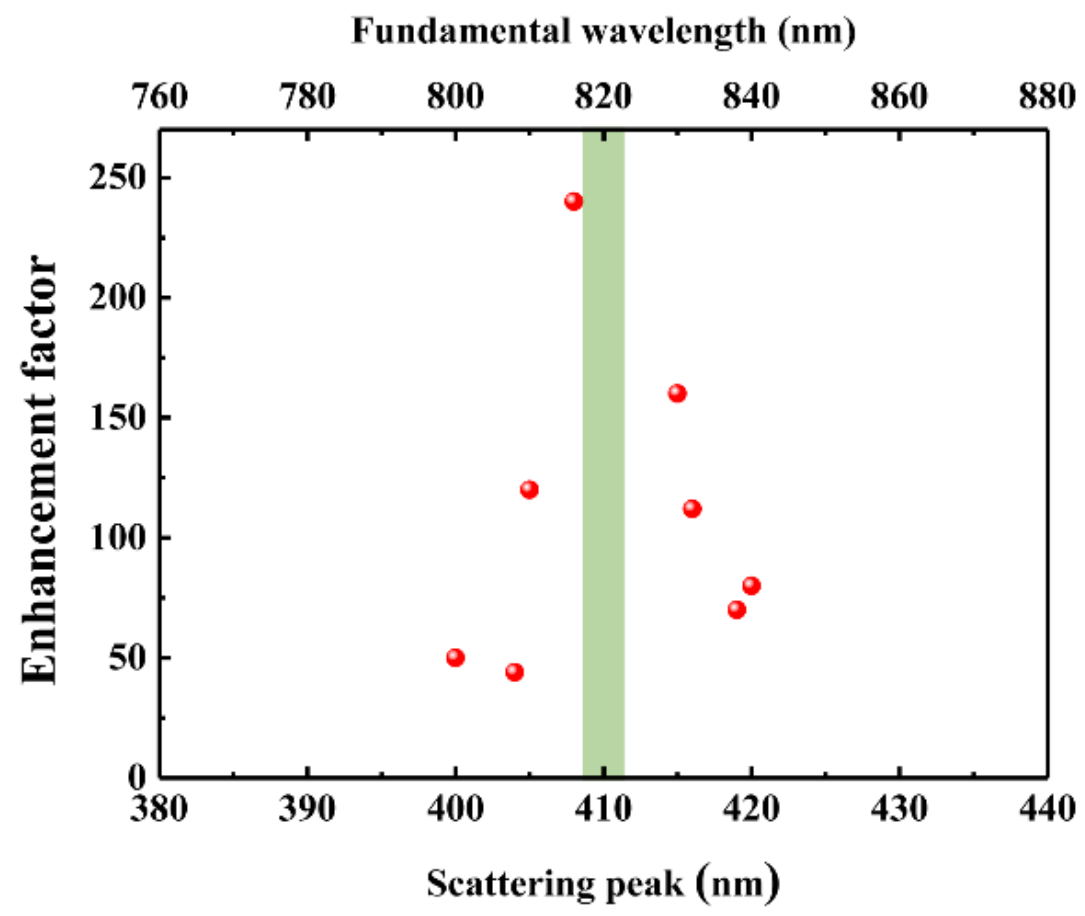

Figure S5. Correlation between SHG enhancement factor (EF) and the scattering peak under the excitation of $820 \mathrm{~nm}$. It indicates that the EF is very sensitive to the scattering peak of plasmonic nanocavity. 

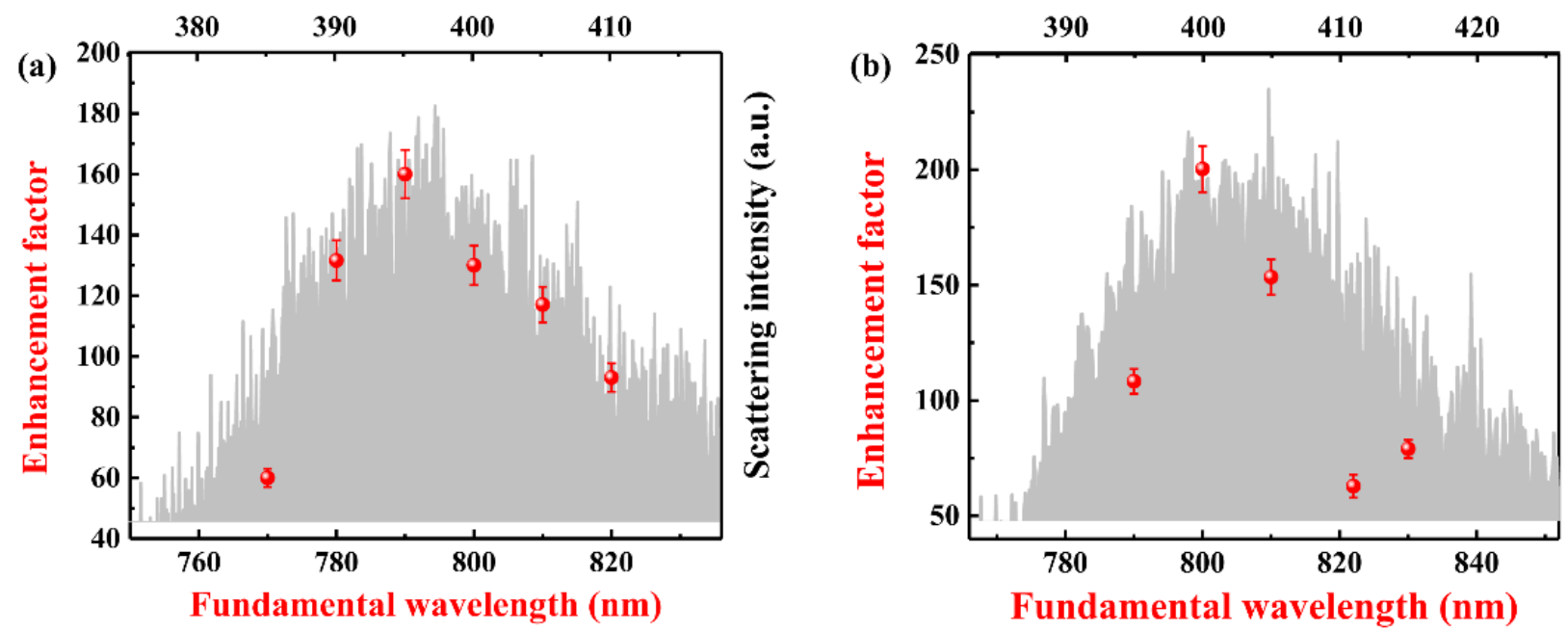

Figure S6. (a, b) Plot of the SHG EF as a function of excitation laser wavelength in the same nanocavity. The red dots represent the experimental data. The normalized linear scattering spectrum of the nanocavity is shown as a gray area. 


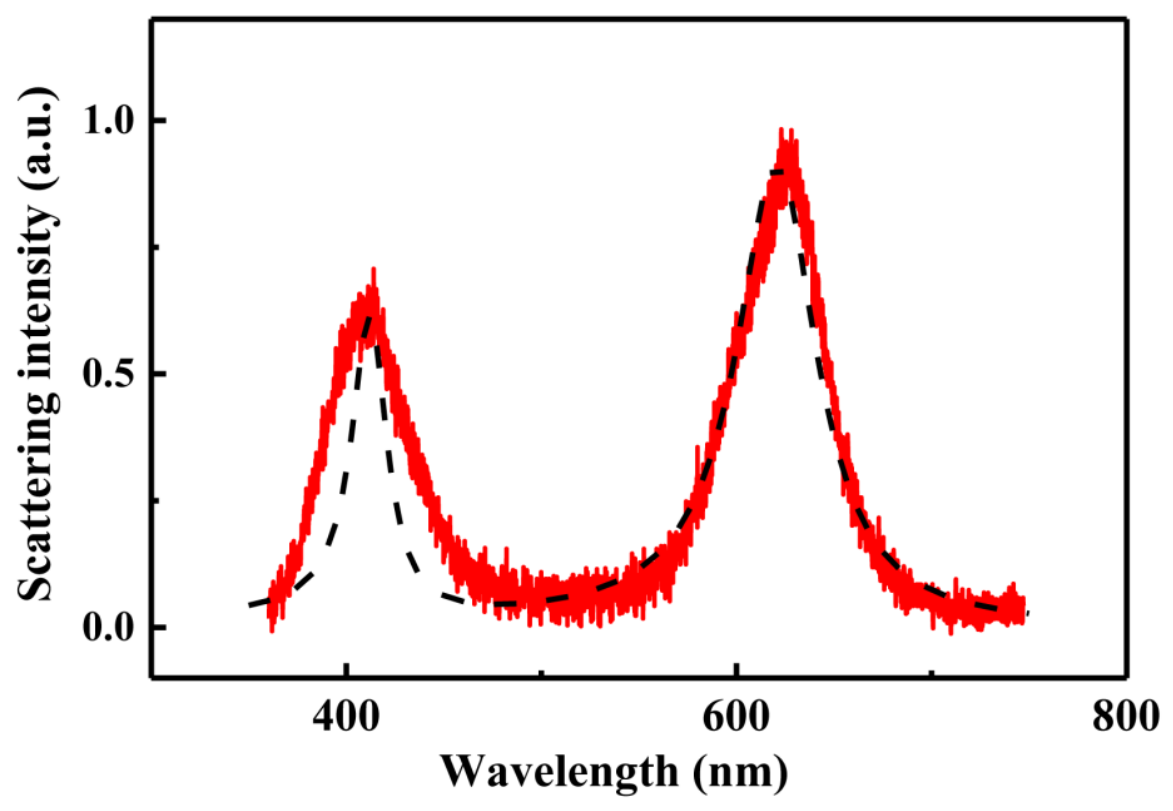

Figure S7. The scattering cross section (AU) for the metallic nanocube alone is plotted as a function of wavelength (nm). The red and black dashed curve represent the experimental data and theoretical calculations for the metallic nanocube, respectively. The SCS spectrum has two peaks located at as $\lambda_{\mathrm{DP}}^{\text {cube }}=620 \mathrm{~nm}$, and $\lambda_{\mathrm{QP}}^{\text {cube }}=410 \mathrm{~nm}$. 


\section{Simulation modeling}

\subsection{Scattering simulation}

The scattering cross-section of individual Ag nanocubes was modeled using the commercial finite element simulation software (COMSOL Multiphysics). A 3-dimensional cuboid domain was created, surrounded by Perfectly Matched Layers (PML, $200 \mathrm{~nm}$ ). An Ag film with a thickness $50 \mathrm{~nm}$ was placed on a silicon substrate with refractive index $n_{\mathrm{b}}=4$. A $9 \mathrm{~nm}$-thick spacer layer $\mathrm{Al}_{2} \mathrm{O}_{3}$ with refractive index $n_{\mathrm{c}}=1.7$ was placed on the $\mathrm{Ag}$ film. ${ }^{2} \mathrm{An} \mathrm{Ag}$ nanocube $(75 \mathrm{~nm}$ in length) was situated on $\mathrm{Al}_{2} \mathrm{O}_{3}$ film with an air gap of thickness $1 \mathrm{~nm}$. The corners of the $\mathrm{Ag}$ nanocube were smoothed with a radius of curvature of $9 \mathrm{~nm}$. The refractive index of metal Ag was used from Johnson \& Christy. ${ }^{3}$ A linear plane wave polarized along $\boldsymbol{y}$ axis, as the excitation source, was normally incident on the $\mathrm{Ag}$ film. The scattered-field formulation was employed, which uses the analytical solution for an incident plane wave in the absence of the Ag nanoucbe as the background. The scattering cross-section is defined as ${ }^{4}$

$$
\sigma_{s c}=\frac{1}{I_{0}} \iint\left(n \cdot S_{s c}\right) d S
$$

Here, $n$ is the normal vector pointing outwards from the Ag nanocube, $S_{s c}$ is the scattered intensity surface (Poynting) vector, and $I_{0}$ is the incident intensity. The integral is taken over the surface of the Ag nanocube.

\subsection{Polarization property of single nanocavities}

To confirm the nanohybrid is independent of the polarization of the excited plane wave. We calculated the electric-field distribution in $x y$ plane with polarized direction at angle $\psi$ of $0,30,45$ and 60 degrees, as shown in Figure S8. The angle $\psi$ was defined as the angle between plane wave polarization direction and $\boldsymbol{y}$ axis. The wavelength of incident plane wave is $410 \mathrm{~nm}$. In general, the 
electric-field distributions in Figure S8 are similar under different angle $\boldsymbol{\psi}$. Furthermore, we calculated the average local-field in volume $\left(75^{*} 75^{*} 0.7 \mathrm{~nm}^{3}\right)$, which is $0.4 \mathrm{~nm}$ below Ag nanocube. For angle $\psi$ of $0,60,45$ and 30 degrees, the corresponding average local field is 3.62, 3.51, 3.45 and 3.51 , respectively. These numerical values are almost unchanged. Therefore, the hybrid nanostructure can be seen as isotropic.
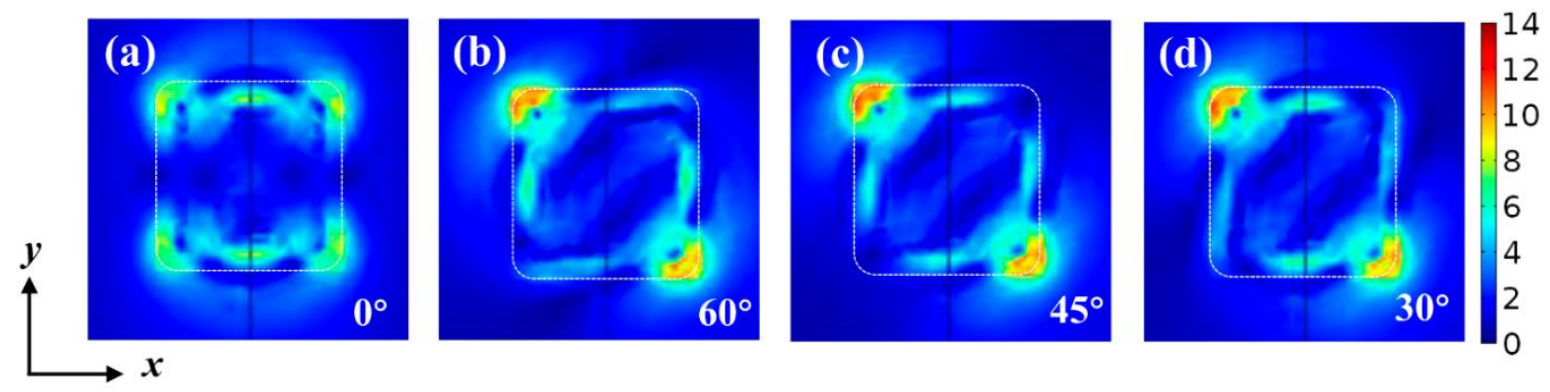

Figure S8. The electric field distribution $\left|\mathbf{E} / \mathbf{E}_{0}\right|$ in $\boldsymbol{x y}$ plane below $\mathrm{Ag}$ nanocube at wavelength of $410 \mathrm{~nm}$. Angle $\psi$ is (a) 0 , (b) 60, (c) 45 and (d) 30 degrees, respectively.

\subsection{Second-harmonic generation (SHG) collection efficiency}

The SHG collection efficiency for the hybrid nanostructure was calculated. A 3-dimensional spherical domain was created and PML condition was as the boundary. A point dipole $(410 \mathrm{~nm})$ with $\boldsymbol{y}$-polarization modeled as the SHG source was placed in the center of Ag nanocube's projection and at the interface between the $\mathrm{Ag}$ nanocube and the $\mathrm{Al}_{2} \mathrm{O}_{3}$ spacer film. The SHG signal was collected with an objective $(100 \times, \mathrm{NA}=0.8)$ in the experiment, so a collection cone with angle $106^{\circ}$ was situated above the point dipole in the simulation. The integrations of far field intensity $\left|\mathbf{E}_{\text {far }}\right|^{2}$ on the end face of the collection cone and on the inner face of PML were calculated to evaluate the collection efficiency. In a comparison, the SHG collection efficiency of a control sample without Ag nanocube was also calculated. The ratio between $\left|\mathbf{E}_{\mathbf{f a r}}\right|^{2}$ with and without $\mathrm{Ag}$ nanocube as the collection efficiency enhancement factor. 


\section{Theoretical Modelling}

We calculate SPP electric-field emitted by the metallic nanocube. We denote the dielectric constant of the metallic nanocube as $\epsilon_{\mathfrak{c}}$, and the dielectric constant for the $\mathrm{WS}_{2}$ layer (the QE) as $\epsilon_{\mathrm{q}}$. We apply a probe field with an amplitude $E_{\mathrm{P}}$, frequency $\omega_{1}$ and wavelength $\lambda$. When the probe field falls on a metallic nanocube, induced dipole and quadrupole moments are produced in the metallic nanocube. The induced dipole and quadrupole moments produce the SPP dipole and the SPP quadrupole electric fields. Let $E_{\mathrm{DP}}^{\text {cube }}$ and $E_{\mathrm{QP}}^{\text {cube }}$ be the electric fields produced by the dipole and quadrupole moments respectively. Here the subscripts DP and QP stand for the dipole and quadrupole components respectively. By solving the Maxwell equations in the static wave approximation, the electric fields produced by the metallic nanocube are calculated as

$$
\begin{array}{ll}
E_{D P}^{\text {cube }}=\left(\Pi_{D P}^{\text {cube }}\right) E_{P}, & \Pi_{D P}^{\text {cube }}=\frac{V_{\text {cube }} \zeta_{D P}^{\text {cube }}}{4 \pi r_{c}^{3}} \\
E_{Q P}^{\text {cube }}=\left(\Pi_{Q P}^{\text {cube }}\right) E_{P}, & \Pi_{Q P}^{\text {cube }}=\frac{V_{\text {cube }}^{5 / 3} \zeta_{Q P}^{\text {cube }}}{4 \pi r_{c}^{5}}
\end{array}
$$

where $r_{\mathrm{c}}$ is the distance between the centre of metallic nanocube and the centre of the QE and $V_{\text {cube }}$ is the volume of the nanocube, Functions $\zeta_{\mathrm{DP}}^{\text {cube }}$ and $\zeta_{\mathrm{QP}}^{\text {cube }}$ are called the polarizability factors, which have the following form

$$
\zeta_{D P}^{\text {cube }}=\frac{\left[\epsilon_{c}-\epsilon_{q}\right]}{3 \xi_{c} \epsilon_{c}+\left(3-3 \xi_{c}\right) \epsilon_{q}}, \quad \zeta_{Q P}^{\text {cube }}=\frac{\left[\epsilon_{c}-\epsilon_{q}\right]}{3 \xi_{c} \epsilon_{c}+(3 / 4)\left(3-3 \xi_{c}\right) \epsilon_{q}}
$$

The parameter $\xi_{\mathrm{c}}$ is called the depolarization factor and depends on the shape of the metallic nanocube.

Its expression is obtained as

$$
\xi_{c}=\frac{V_{c u b e}}{2} \int_{0}^{\infty} \frac{d s}{\left(s+L_{c x}^{2}\right)^{3 / 2}\left(s+L_{c y}^{2}\right)^{1 / 2}\left(s+L_{c z}^{2}\right)^{1 / 2}}
$$


where $L_{\mathrm{cx}}, L_{\mathrm{cy}}$ and $L_{\mathrm{cz}}$ are the length, width and thickness of the metallic nanocube respectively.

Next, we calculated the SPP field produced by the metallic thin film, with a thickness of $80 \mathrm{~nm}$ and an infinite width and length. The half width of the metallic field is denoted as $W_{\mathrm{f}}$ and the thickness of the spacer layer is denoted as $t_{\mathrm{s}}$. When a probe field is applied to the system, with an amplitude denoted as $E_{\mathrm{P}}$, dipoles are created in the metallic thin film. SPPs are present at the interface between the metallic film and the spacer layer due to the dipole moments. We denote the dielectric constant of the metallic Ag film as $\epsilon_{\mathrm{f}}$, and the dielectric constant of the spacer layer is denoted as $\epsilon_{\mathrm{s}}$. Note that the metallic nanocube and the metallic film are both made from $\mathrm{Ag}$, so they have the same dielectric constant as the nanocube i.e. $\epsilon_{\mathrm{f}}=\epsilon_{\mathrm{c}}$. We can not apply the quasi-static approximation to the metallic film since its size is greater than the wavelength of the probe laser. Due to this we follow the method of reference. ${ }^{5}$ We found the expression of the SPP field as the following:

$$
E_{D P}^{f i l m}=\left(\Pi_{D P}^{f i l m}\right) E_{P}, \quad \Pi_{D P}^{f i l m}=\zeta_{D P}^{f i l m}(\omega) \exp \left(-k_{q} r_{f}\right), \quad k_{q}=\frac{\omega_{1}}{c} \sqrt{\left(\zeta_{D P}^{f i l m}-\epsilon_{q}\right)}
$$

where $k_{\mathrm{q}}$ is the SPP wave-vector propagating in the QE layer, and $r_{f}$ is the distance between the middle of the metallic film and the location of the QE. Here, $\zeta_{\mathrm{DP}}^{\text {film }}$ is the polarization factor for the film and it is found as

$$
\zeta_{D P}^{\text {film }}=\frac{\epsilon_{s f} \in_{q}}{\epsilon_{s f}+\epsilon_{q}}, \quad \in_{s f}=\in_{f} \frac{\left(W_{f}+t_{s}\right)+W_{f}\left(\frac{\epsilon_{f} \epsilon_{s}}{\epsilon_{f}+\epsilon_{s}}\right)}{\left(W_{f}+t_{s}\right)-W_{f}\left(\frac{\epsilon_{f} \epsilon_{s}}{\epsilon_{f}+\epsilon_{s}}\right)}
$$

We will use the following expression for the dielectric constant of the metallic nanocube

$$
\epsilon_{c}=\epsilon_{\infty}\left(1-\frac{\omega_{p}^{2}}{\omega_{1}^{2}+i \omega_{1} \gamma_{c}}\right)
$$


where $\omega_{\mathrm{p}}$ and $\epsilon_{\infty}$ are the plasmon frequency and the dielectric constant of metal when light frequency is very large, respectively. The parameter $\gamma_{c}$ is the decay rate and it represents the heat energy loss in a metallic nanocube.

Note that the real part of $\epsilon_{\mathrm{c}}$ has negative value when $\omega<\omega_{\mathrm{p}}$. The polarizability factor $\zeta_{\mathrm{DP}}^{\text {cube }}, \zeta_{\mathrm{QP}}^{\text {cube }}$ and $\zeta_{\mathrm{DP}}^{\text {film }}$ have $\epsilon_{\mathrm{c}}$ in the denominator which becomes zero at certain values of the frequency. This frequency is called SPP frequency/wavelength. Using equation (3), the SPP resonances due to the metallic nanocube are calculated from the polarization factor $\operatorname{Im}\left(\zeta_{\mathrm{DP}}^{\text {cube }}\right)$ and $\operatorname{Im}\left(\zeta_{\mathrm{QP}}^{\mathrm{cube}}\right)$. When the denominators of the polarization factors, $\operatorname{Im}\left(\zeta_{\mathrm{DP}}^{\text {cube }}\right)$ and $\operatorname{Im}\left(\zeta_{\mathrm{QP}}^{\text {cube }}\right)$, become zero for $\omega=\omega_{\mathrm{DP}}^{\text {cube }}$ and $\omega$ $=\omega_{\mathrm{QP}}^{\text {cube }}$, the dipole and quadrupole SPP electric fields produced by the metallic nanocube have huge values. These frequencies $\omega_{\mathrm{DP}}^{\text {cube }}$ and $\omega_{\mathrm{QP}}^{\text {cube }}$ are called SPP resonances. It is important to note from equation (3) that the denominator of the dipole polarization factor $\operatorname{Im}\left(\zeta_{\mathrm{DP}}^{\mathrm{cube}}\right)$ is different than the denominator of the quadrupole polarization factor $\operatorname{Im}\left(\zeta_{\mathrm{QP}}^{\text {cube }}\right)$. Due to these differences, $\omega_{\mathrm{DP}}^{\text {cube }}$, and $\omega_{\mathrm{QP}}^{\text {cube }}$, have different values. Similarly, we can also calculate the SPP resonance frequency $\omega_{\mathrm{sp}}^{\text {film }}$ for the metallic film. The SPP electric field produced by the metallic film has also huge value at $\omega=\omega_{\mathrm{sp}}^{\text {film }}$.

Additionally, we calculated the scattering cross section (SCS) due to the one-photon process for the Ag nanocube only. We have shown that the nanocube emits the SPP dipole and dipole fields. Following the method of reference, ${ }^{6-7}$ we have evaluated the SCS for the Ag-nanocube only and found it to be

$$
\sigma_{\text {scatt }}=\frac{\omega_{1}^{4} \in_{b}^{2}}{6 \pi c^{4}}\left|V_{\text {cube }} \zeta_{D P}^{\text {cube }}\right|^{2}+\frac{\omega_{1}^{6} \in_{b}^{2}}{9 \pi c^{6}}\left|V_{\text {cube }}^{4 / 3} \zeta_{Q P}^{\text {cube }}\right|^{2}
$$

Note that the SCS depends on $\zeta_{\mathrm{DP}}^{\text {cube }}$ and $\zeta_{\mathrm{QP}}^{\text {cube }}$ which are the dipole and quadrupole polarization parameters of the metallic nanocube. 
Using our theory we calculated the SCS intesnity and were able to compare the resutls with the the experimental data for the hybrid structure fabricated from nanocavity and QEs. In the nanohybrid,

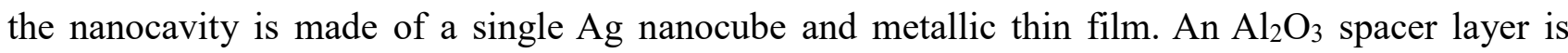
deposited on the top of the metallic thin film. The monolayer $\mathrm{WS}_{2}$ is deposited between the space layer and the Ag nanocube. The thickness of $\mathrm{WS}_{2}$ layer is about $0.7 \mathrm{~nm}$. The dielectric constant of $\mathrm{WS}_{2}$ is taken as $\epsilon_{\mathrm{q}}=6.2$. The thickness of Ag film is $80 \mathrm{~nm}$ and its length and width are infinite. The length of each side of the Ag nanocube is $\sim 75 \mathrm{~nm}$. Using these physical parameters, we have calculated the dipole and the quadrupole SPP resonance wavelengths and found as $\lambda_{\mathrm{DP}}^{\text {cube }}=620 \mathrm{~nm}$, and $\lambda_{\mathrm{QP}}^{\text {cube }}=410$ $\mathrm{nm}$, respectively. The SPP resonance wavelength due to the metallic film is also calculated and found as $\lambda_{\mathrm{sp}}^{\text {film }}=120 \mathrm{~nm}$. Note that due to the wavelength of dipole SPP resonance and Ag film SPP resonance is far away from the SHG wavelength, the SHG intensity is mainly affected by the quadrupole SPP presented in Ag nanocubes.

The SCS for an Ag nanocube located inside the nanocavity was measured by using a white light illumination, which is a typical way of testing linear optical properties. The measured SCS data was due to the one-photon process as opposed to a two-photon process. The results are plotted in Figure S7 as a function of wavelength. The experimental data are plotted as red curve and the black dashed line is plotted for theoretical simulations. Note that the SCS spectrum has two peaks located at $\lambda_{\mathrm{QP}}^{\text {cube }}=$ $410 \mathrm{~nm}$ and $\lambda_{\mathrm{DP}}^{\text {cube }}=620 \mathrm{~nm}$. The first peak corresponds to the quadrupole SPP resonance and the second peak corresponds to the dipole SPP resonance. The intensity of the second peak is higher than the first peak because the dipole SPP coupling is stronger than the quadrupole SPP coupling. Note that a good agreement between our theory and the experiment was found on a qualitative basis but not on a quantitative basis.

\section{Estimation of the SHG conversion efficiency}


The SHG conversion efficiency, $\delta$, are estimated based on the ratio of peak values between the SHG signal and the pumping laser, $I_{\mathrm{sHG}} / I_{\text {pump. }}$ It can be obtained from the measured spectrum shown in Figure S9. It is worth noting that a short pass filter $(720 \mathrm{~nm}$, Semrock, FF01-720/SP-25) was placed in front of the spectrometer. The short pass filter acts as an attenuator for the pumping laser with an attenuation factor $\zeta$ of $10^{-9}$. Since both the signals were collected through a series of reflective mirror, and lens, the SHG conversion efficiency should be modified by considering some other factors:

$$
\delta=\frac{G_{\text {pump }}}{G_{\mathrm{SHG}}} \cdot \frac{Q_{\text {pump }}}{Q_{\mathrm{SHG}}} \cdot\left(\frac{R_{\mathrm{pump}}}{R_{\mathrm{SHG}}}\right)^{m} \cdot\left(\frac{T_{\text {pump }}}{T_{\mathrm{SHG}}}\right)^{n} \cdot \frac{\zeta \cdot I_{\mathrm{SHG}}}{I_{\mathrm{pump}}}
$$

where $G_{\text {pump }}$ and $G_{\mathrm{SHG}}$ are the grating efficiencies for the wavelength of the fundamental and SHG wave; $Q_{\text {pump }}$ and $Q_{\mathrm{SHG}}$ are the quantum efficiencies of the $\mathrm{CCD}$ at the wavelength of the fundamental and SHG wave. $R_{\text {pump }}$ and $R_{\mathrm{SHG}}$ are the reflectance of $\mathrm{Ag}$ mirror for the fundamental and SHG wave. $T_{\text {pump }}$ and $T_{\mathrm{SHG}}$ are the transmittance of lens for the fundamental and SHG wave. $m$ and $n$ are the numbers of Ag mirror and lens between the sample and the spectrometer, respectively. In our experimental setup, $m$ and $n$ are both 3 . The above ratios can be obtained in the product manuals listed in Table S1. According to the Equation 9, the SHG conversion efficiency, $\delta$, in monolayer $\mathrm{WS}_{2}$ on $\mathrm{Al}_{2} \mathrm{O}_{3}-\mathrm{Ag}$ film is estimated to be $7 \times 10^{-9}$ under the pumping power of $0.8 \mathrm{~mW}$. And the $\mathrm{SHG}$ conversion efficiency, $\delta$, is calculated to be $2 \times 10^{-6}$ for the $\mathrm{WS}_{2}$ in NPoM structure.

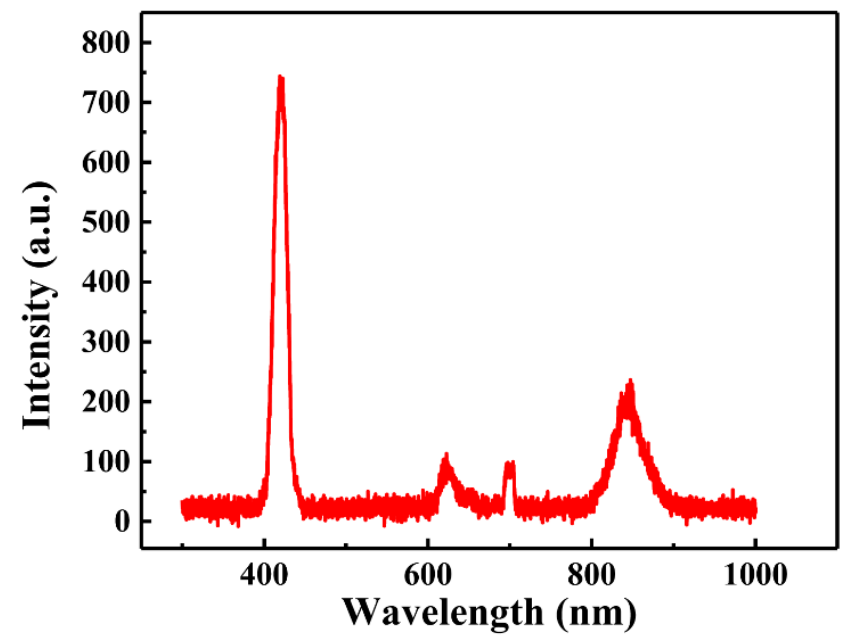

Figure S9. Spectrum of the collected SHG signal and the corresponding pumping laser. The signals at $620 \mathrm{~nm}$ 
and $700 \mathrm{~nm}$ are the two-photon-absorption-induced photoluminescence from monolayer $\mathrm{WS}_{2}$ and the accompanying light of the pumping laser.

Table S1. The ratio values for the estimation of SHG conversion efficiency.

\begin{tabular}{|l|l|l|l|}
\hline$G_{\text {pump }} / G_{\mathrm{SHG}}$ & $Q_{\text {pump }} / Q_{\mathrm{SHG}}$ & $R_{\text {pump }} / R_{\mathrm{SHG}}$ & $T_{\text {pump }} / T_{\mathrm{SHG}}$ \\
\hline $48 \% / 47 \%$ & $92 \% / 45 \%$ & $97 \% / 95.6 \%$ & $94.9 \% / 99.4 \%$ \\
\hline
\end{tabular}

Several SHG enhancement results from hybrid structures are listed in Table S2. Most SHG enhancement mechanism is based on fundamental resonance. Although the enhancement factor in our work is not the highest, harmonic resonance as a novel SHG enhancement mechanism is presented, which has potential applications in hybrid nonlinear structures.

Table S2. SHG enhancement experiments by using versatile plasmonic effects.

\begin{tabular}{|c|c|c|c|c|}
\hline Material & Structure & $\begin{array}{l}\text { Conversion } \\
\text { efficiency }\end{array}$ & $\begin{array}{l}\text { Enhancement } \\
\text { factor }\end{array}$ & References \\
\hline $\mathrm{BaTiO}_{3} / \mathrm{Au}$ & Nanosphere cavity & - & $\sim 500$ & $\begin{array}{l}\text { Phys. Rev. } \\
\text { Lett. 2010, } \\
\text { 104, } 207402 .\end{array}$ \\
\hline $\mathrm{ZnO} / \mathrm{Au}$ & $\begin{array}{c}\text { Single Nanowire/Plasmonic } \\
\text { Oligomer }\end{array}$ & $\sim 3 \times 10^{-7}$ & $\sim 1700$ & $\begin{array}{l}\text { Nano Lett. } \\
\text { 2014, 14, } \\
6660-6665 .\end{array}$ \\
\hline CdS/Ag & $\begin{array}{c}\text { Single Nanowire/Ag WGM } \\
\text { cavity }\end{array}$ & $\sim 3 \times 10^{-6}$ & $>1000$ & $\begin{array}{c}\text { Nat. } \\
\text { Commun. } \\
2014,5,5432 .\end{array}$ \\
\hline GaAs/Au & Nanowire/Au disks & - & $\sim 20$ & $\begin{array}{l}\text { Nano Lett. } \\
\text { 2014, 14, } \\
2271-2278 .\end{array}$ \\
\hline CdS/Au & $\begin{array}{l}\text { Single Nanowire/ } \mathrm{SiO}_{2} / \mathrm{Au} \\
\text { film }\end{array}$ & $\sim 2 \times 10^{-8}$ & $\sim 1000$ & $\begin{array}{l}\text { ACS Nano } \\
2015,9 \\
5018-5026 .\end{array}$ \\
\hline $\mathrm{LiNbO}_{3} / \mathrm{Au}$ & Nanoring resonator & - & $\sim 20$ & $\begin{array}{l}\text { Nano Lett. } \\
2015,15, \\
1025-1030 .\end{array}$ \\
\hline
\end{tabular}




\begin{tabular}{|c|c|c|c|c|}
\hline $\mathrm{MoS}_{2} / \mathrm{Ag}$ & $\begin{array}{c}\text { Monolayer } \mathrm{MoS}_{2} / \mathrm{Ag} \\
\text { nanowire }\end{array}$ & $\sim 2.19 \times 10^{-8}$ & - & $\begin{array}{c}\text { Nano Lett. } \\
\text { 2017, 17, } \\
\text { 7803-7808. }\end{array}$ \\
\hline $\mathbf{W S}_{2} / \mathbf{A g}$ & $\begin{array}{c}\text { Monolayer } \mathrm{MoS}_{2} / \mathrm{Ag} \\
\text { nanogroove grating }\end{array}$ & $\sim 2 \times 10^{-5}$ & $\sim 400$ & $\begin{array}{c}\text { Laser } \\
\text { Photonics } \\
\text { Rev. 2018, 12, } \\
1800188 .\end{array}$ \\
\hline $\mathbf{W S e}_{2} / \mathrm{Au}$ & $\begin{array}{c}\text { Monolayer } \mathrm{WSe}_{2} / \mathrm{Au} \\
\text { trenches }\end{array}$ & - & $\sim 7000$ & $\begin{array}{c}\text { ACS Nano } \\
2018,12 \\
1859-1867 .\end{array}$ \\
\hline CdSe/Au & Single nanowire/Au film & $\sim 4 \times 10^{-5}$ & - & $\begin{array}{l}\text { Nano Lett. } \\
\text { 2019, 19, } \\
\text { 3838-3845. }\end{array}$ \\
\hline $\mathrm{WS}_{2} / \mathbf{A g}$ & Monolayer $\mathrm{WS}_{2} / \mathrm{NPoM}$ & $\sim 2 \times 10^{-6}$ & $\sim 280$ & This work \\
\hline
\end{tabular}

\section{References}

(1) Woods, J. M.; Jung, Y.; Xie, Y.; Liu, W.; Liu, Y.; Wang, H.; Cha, J. J. One-step synthesis of $\mathrm{MoS}_{2} / \mathrm{WS}_{2}$ layered heterostructures and catalytic activity of defective transition metal dichalcogenide films. ACS Nano 2016, 10, 2004-9.

(2) Boidin, R.; Halenkovič, T.; Nazabal, V.; Beneš, L.; Němec, P. Pulsed laser deposited alumina thin films. Ceram. Int. 2016, 42, 1177-1182.

(3) Johnson, P. B.; Christy, R. W. Optical constants of the noble metals. Phys. Rev. B 1972, 6, 43704379.

(4) https://cn.comsol.com/models.

(5) Singh, M. R., Electronic, photonic, polaritonic and plasmonic materials. Wiley Custom, Toronto: 2014.

(6) Sarid, D.; Challener, W. A., Modern introduction to surface plasmons: Theory, mathematica modeling, and applications. Cambridge University Press: 2010.

(7) Novotny, L.; Hecht, B., Principles of nano-optics. Cambridge university press: 2012. 